\author{
Universidade de São Paulo \\ Escola de Engenharia de São Carlos \\ Instituto de Física de Sã Carlos \\ Instituto de Química de São Carlos
}

\title{
A DENSIDADE APARENTE COMO ESTIMADOR DE PROPRIEDADES DE RESISTÊNCIA E RIGIDEZ DA MADEIRA
}

\section{Fabricio Moura Dias}

Dissertação apresentada à Área Interunidades em ciência e Engenharia de Materiais, Campus de São Carlos, da Universidade de São Paulo, como parte dos requisitos para obtenção do título de Mestre em Ciência e Engenharia de Materiais

ORIENTADOR: Prof. Tit. Francisco Antonio Rocco Lahr

São Carlos 
"A pessoa inteira é aquela que estabelece um contato significativo e profundo com o mundo à sua volta. Ela não só escuta a si mesma, como também às vozes de seu mundo. A extensão de sua própria experiência é infinitamente multiplicada pela empatia que sente em relação aos outros."

John Powell 
A meus pais, José e Laura, com quem vivo minha mais profunda relação de ajuda. A meus irmãos, Fábio, Fabíola e Fabiano, a um só tempo, os incentivadores mais efetivos, os ajudados mais ternos.

Ao mestre Manoel, que me ajudou a perder o medo de perder, condição fundamental para ganhar. 


\section{AGRADECIMENTOS}

Ao professor Francisco Antonio Rocco Lahr - excelente orientador e grande amigo.

À minha família e amigos - sempre úteis e efetivos. Nos momentos difíceis, sentaram-se ao meu lado e mesmo em silêncio, ajudaram a transformar as incertezas $e$ inseguranças em sentimentos valorosos $e$ positivos.

À Marcela, Geraldo e Nayara, que me acolheram com muito carinho.

À Fátima, tantas vezes, grande incentivadora.

Ao Bragatto e Roberto, pela constante ajuda.

Ao Conselho Nacional de Desenvolvimento Científico e Tecnológico - CNPq, pela bolsa de estudos concedida.

A todos os colegas, professores e funcionários do Laboratório de Madeiras e Estruturas de Madeira e da Área Interunidades, Universidade de São Paulo, pelo carinho e colaboração. 


\section{SUMÁRIO}

LISTA DE FIGURAS Tix

LISTA DE TABELAS Xvi

LISTA DE ABREVIATURAS E SIGLAS Xx

LISTA DE SÍMBOLOS Xxi

RESUMO Xxiii

ABSTRACT Xxiv

1. INTRODUÇÃO 1

2. REVISÃO BIBLIOGRÁFICA 3

2.1. GENERALIDADES 3

2.2. ESTRUTURA E COMPOSIÇÃO QUÍMICA DA MADEIRA 4

2.3. DENSIDADE 10

2.3.1. DENSIDADE BÁSICA 11

2.3.2. DENSIDADE APARENTE 12

2.4. UMIDADE E SUA INFLUÊNCIA SOBRE A DENSIDADE 14

2.5. RETRATIBILIDADE E SUA RELAÇÃO COM A DENSIDADE 18

2.6. RELAÇÕES ENTRE DENSIDADE E CARACTERÍSTICAS ANATÔMICAS E QUÍMICAS DA MADEIRA 19

2.7. RELAÇÕES DAS PROPRIEDADES DE RESISTÊNCIA E RIGIDEZ DA MADEIRA COM A DENSIDADE 22

2.7.1. RESISTÊNCIA À COMPRESSÃO PARALELA ÀS FIBRAS 24

2.7.2. RESISTÊNCIA À TRAÇÃO PARALELA ÀS FIBRAS 27

2.7.3. RESISTÊNCIA À TRAÇÃO NORMAL ÀS FIBRAS 28

2.7.4. RESISTÊNCIA AO CISALHAMENTO PARALELO ÀS 29 FIBRAS 
2.7.5. RESISTÊNCIA CONVENCIONAL NO ENSAIO DE FLEXÃO ESTÁTICA

2.7.6. MÓDULO DE ELASTICIDADE 32

2.7.7. DUREZA 33

2.7.8. TENACIDADE 35

2.8. CONSIDERAÇÕES SOBRE A REVISÃO DA LITERATURA 38

3. MATERIAIS E MÉTODOS 40

3.1. BREVE DESCRIÇÃO DAS ESPÉCIES UTILIZADAS 41

3.1.1. ANGELIM-AMARGOSO 41

3.1.2. ANGELIM-ARAROBA 42

3.1.3. ANGELIM-FERRO 42

3.1.4. ANGELIM-PEDRA-VERDADEIRO 42

3.1.5. ANGELIM-PEDRA 43

3.1.6. ANGELIM-SAIA 43

3.1.7. ANGICO-PRETO 43

3.1.8. BRANQUILHO 44

3.1.9. CAFEARANA 44

3.1.10. CANAFÍSTULA 44

3.1.11. CASCA-GROSSA 45

3.1.12. CASTELO 45

3.1.13. CATANUDO 45

3.1.14. CEDRO-AMARGO 46

3.1.15. CEDRO-DOCE 46

3.1.16. CEDRORANA 46

3.1.17. CHAMPAGNE 47

3.1.18. COPAÍBA 47

3.1.19. CUPIÚBA 47

3.1.20. CUTIÚBA 48

3.1.21. GARAPA 48

3.1.22. GOIABÃO 48

3.1.23. GUAIÇARA 49

3.1.24. GUARUCAIA 49 
3.1.25. IPÊ 49

3.1.26. ITAÚBA 50

3.1.27. JATOBÁ $\quad 50$

3.1.28. LOURO-PRETO 51

3.1.29. MAÇARANDUBA 51

3.1.30. MANDIOQUEIRA 51

3.1.31. OITICICA-AMARELA 52

3.1.32. OIUCHU 52

3.1.33. PARINARI 52

3.1.34. PIOLHO 53

3.1.35. QUARUBARANA 53

3.1.36. RABO-DE-ARRAIA 53

3.1.37. SUCUPIRA 54

3.1.38. TACHI 54

3.1.39. TATAJUBA 54

3.1.40. UMIRANA 55

3.2. PROCEDIMENTOS PARA ANÁLISE ESTATÍSTICA 55

3.3. ANÁLISE ESTATÍSTICA 57

4. RESULTADOS E DISCUSSÕES 61

4.1. REGRESSÕES PARA OBTENÇÃO DAS RELAÇÕES ENTRE A DENSIDADE APARENTE E AS PROPRIEDADES DE RESISTÊNCIA E RIGIDEZ DA MADEIRA 61

4.1.1. RELAÇÃO DA DENSIDADE APARENTE COM DEMAIS PROPRIEDADES 76

4.2. APRESENTAÇÃO DAS CLASSES DE RESISTÊNCIA 77

4.3. AFERIÇÃO DAS EXPRESSÕES UTILIZADAS PARA CARACTERIZAÇÃO SIMPLIFICADA DAS RESISTÊNCIAS DA MADEIRA

5. CONCLUSÕES 86 
$\boldsymbol{A N E X O}$ - A) Tabelas de valores das propriedades de resistência e rigidez da madeira para as espécies estudadas.

B) Gráficos representativos dos ajustes de linha para as variáveis analisadas.

6. REFERÊNCIAS BIBLIOGRÁFICAS 


\section{LISTA DE FIGURAS}

FIGURA 1 - Representação da característica anisotrópica da madeira. $\quad 4$

FIGURA 2 - Estrutura celular da madeira do grupo dicotiledônea. 5

FIGURA 3 - Estrutura simplificada das camadas celulares da madeira com orientações das fibras.

FIGURA 4 - Representação da unidade de celubiose e extremidades das cadeias de celulose.

FIGURA 5 - Diagrama de Kollmann: representa a variação da densidade aparente com o teor de umidade.

FIGURA 6 - Influência do teor de umidade sobre a densidade aparente, para a cupiúba (Goupia glabra).

FIGURA 7 - Influência do teor de umidade sobre a densidade aparente, para o jatobá (Hymenaea stilbocarpa).

FIGURA 8 - Relação entre a resistência à compressão paralela, a densidade e a umidade para o Pinho do Paraná.

FIGURA 9 - Influência da umidade e da densidade sobre a resistência à compressão paralela às fibras.

FIGURA 10- Diagrama da relação da densidade com a resistência à compressão paralela às fibras.

FIGURA 11- Diagrama representativo da relação da densidade básica com a resistência à tração paralela às fibras

FIGURA 12 - Variação da resistência da madeira à tração normal às fibras em função da densidade aparente.

FIGURA 13- Influência da umidade sobre a resistência à flexão, para diversos níveis de densidade da espécie jatobá. 
FIGURA 14 - Influência da densidade sobre a resistência à flexão, para diversos níveis de umidade da espécie jatobá.

FIGURA 15- Representação gráfica da variação do módulo de elasticidade em função da variação da densidade da madeira.

FIGURA 16 - Efeito da densidade sobre a dureza da madeira.

FIGURA 17 - Efeito da densidade aparente sobre a tenacidade na madeira. 36

FIGURA 18 - Efeito da densidade sobre a tenacidade para as madeiras pine, spruce, beech e oak.

FIGURA 19 - Diagrama de resíduos sem tendenciosidade (resíduos oscilam em torno da média zero).

FIGURA 20 - Diagrama de resíduos com tendenciosidade (resíduos apresentam variação funcional).

FIGURA 21 - Diagrama de resíduos com tendenciosidade (resíduos apresentam hetereogeneidade de variâncias).

FIGURA 22 - Gráfico de resíduos em função da densidade aparente para a regressão entre a densidade aparente e a resistência à compressão paralela às fibras - agrupamento dos valores individuais.

FIGURA 23 - Gráfico de resíduos em função da densidade aparente para a regressão entre a densidade aparente e a resistência à tração paralela às fibras - agrupamento dos valores individuais.

FIGURA 24 - Gráfico de resíduos em função da densidade aparente para a regressão entre a densidade aparente e a resistência ao cisalhamento paralelo às fibras - agrupamento dos valores individuais.

FIGURA 25 - Gráfico de resíduos em função da densidade aparente para a regressão entre a densidade aparente e a resistência convencional no ensaio de flexão estática - agrupamento dos valores individuais. 
FIGURA 26 - Gráfico de resíduos em função da densidade aparente para a regressão entre a densidade aparente e o módulo de elasticidade na compressão paralela às fibras - agrupamento dos valores individuais.

FIGURA 27 - Gráfico de resíduos em função da densidade aparente para a regressão entre a densidade aparente e o módulo de elasticidade na tração paralela às fibras - agrupamento dos valores individuais.

FIGURA 28 - Gráfico de resíduos em função da densidade aparente para a regressão entre a densidade aparente e o módulo de elasticidade na flexão estática - agrupamento dos valores individuais.

FIGURA 29 - Gráfico de resíduos em função da densidade aparente para a regressão entre a densidade aparente e a dureza paralela às fibras - agrupamento dos valores individuais.

FIGURA 30 - Gráfico de resíduos em função da densidade aparente para a regressão entre a densidade aparente e a dureza normal às fibras - agrupamento dos valores individuais.

FIGURA 31 - Gráfico de resíduos em função da densidade aparente para a regressão entre a densidade aparente e a tenacidade agrupamento dos valores individuais.

FIGURA 32 - Gráfico de resíduos em função da densidade aparente para a regressão entre a densidade aparente e a resistência à compressão paralela às fibras - agrupamento dos valores médios para todas espécies.

FIGURA 33 - Gráfico de resíduos em função da densidade aparente para a regressão entre a densidade aparente e a resistência à tração paralela às fibras - agrupamento dos valores médios para todas espécies. 
FIGURA 34 - Gráfico de resíduos em função da densidade aparente para a regressão entre a densidade aparente e a resistência ao cisalhamento paralelo às fibras - agrupamento dos valores médios para todas espécies.

FIGURA 35 - Gráfico de resíduos em função da densidade aparente para a regressão entre a densidade aparente e a resistência convencional no ensaio de flexão estática - agrupamento dos valores médios para todas espécies.

FIGURA 36 - Gráfico de resíduos em função da densidade aparente para a regressão entre a densidade aparente e o módulo de elasticidade na compressão paralela às fibras - agrupamento dos valores médios para todas espécies.

FIGURA 37 - Gráfico de resíduos em função da densidade aparente para a regressão entre a densidade aparente e o módulo de elasticidade na tração paralela às fibras - agrupamento dos valores médios para todas espécies.

FIGURA 38 - Gráfico de resíduos em função da densidade aparente para a regressão entre a densidade aparente e o módulo de elasticidade na flexão estática - agrupamento dos valores médios para todas espécies.

FIGURA 39 - Gráfico de resíduos em função da densidade aparente para a regressão entre a densidade aparente e a dureza paralela às fibras - agrupamento dos valores médios para todas espécies.

FIGURA 40 - Gráfico de resíduos em função da densidade aparente para a regressão entre a densidade aparente e a dureza normal às fibras - agrupamento dos valores médios para todas espécies.

FIGURA 41 - Gráfico de resíduos em função da densidade aparente para a regressão entre a densidade aparente e a tenacidade agrupamento dos valores médios para todas espécies. 
FIGURA 42 - Gráfico de ajuste de linha para a relação entre a densidade aparente e a resistência à compressão paralela às fibras agrupamento dos valores individuais.

FIGURA 43 - Gráfico de ajuste de linha para a relação entre a densidade aparente e a resistência à tração paralela às fibras agrupamento dos valores individuais.

FIGURA 44 - Gráfico de ajuste de linha para a relação entre a densidade aparente e a resistência ao cisalhamento paralelo às fibras agrupamento dos valores individuais.

FIGURA 45 - Gráfico de ajuste de linha para a relação entre a densidade aparente e a resistência convencional no ensaio de flexão estática - agrupamento dos valores individuais.

FIGURA 46 - Gráfico de ajuste de linha para a relação entre a densidade aparente e o módulo de elasticidade na compressão paralela às fibras - agrupamento dos valores individuais.

FIGURA 47 - Gráfico de ajuste de linha para a relação entre a densidade aparente e o módulo de elasticidade na tração paralela às fibras - agrupamento dos valores individuais.

FIGURA 48 - Gráfico de ajuste de linha para a relação entre a densidade aparente e o módulo de elasticidade na flexão estática agrupamento dos valores individuais.

FIGURA 49 - Gráfico de ajuste de linha para a relação entre a densidade aparente e a dureza paralela às fibras - agrupamento dos valores individuais.

FIGURA 50 - Gráfico de ajuste de linha para a relação entre a densidade aparente e a dureza normal às fibras - agrupamento dos valores individuais.

FIGURA 51 - Gráfico de ajuste de linha para a relação entre a densidade aparente e a tenacidade - agrupamento dos valores individuais. 
FIGURA 52 - Gráfico de ajuste de linha para a relação entre a densidade aparente e a resistência à compressão paralela às fibras agrupamento dos valores médios para todas espécies.

FIGURA 53 - Gráfico de ajuste de linha para a relação entre a densidade aparente e a resistência à tração paralela às fibras agrupamento dos valores médios para todas espécies.

FIGURA 54 - Gráfico de ajuste de linha para a relação entre a densidade aparente e a resistência ao cisalhamento paralelo às fibras agrupamento dos valores médios para todas espécies.

FIGURA 55 - Gráfico de ajuste de linha para a relação entre a densidade aparente e a resistência convencional no ensaio de flexão estática - agrupamento dos valores médios para todas espécies.

FIGURA 56 - Gráfico de ajuste de linha para a relação entre a densidade aparente e o módulo de elasticidade na compressão paralela às fibras - agrupamento dos valores médios para todas espécies.

FIGURA 57 - Gráfico de ajuste de linha para a relação entre a densidade aparente e o módulo de elasticidade na tração paralela às fibras - agrupamento dos valores médios para todas espécies.

FIGURA 58 - Gráfico de ajuste de linha para a relação entre a densidade aparente e o módulo de elasticidade na flexão estática agrupamento dos valores médios para todas espécies.

FIGURA 59 - Gráfico de ajuste de linha para a relação entre a densidade aparente e a dureza paralela às fibras - agrupamento dos valores médios para todas espécies.

FIGURA 60 - Gráfico de ajuste de linha para a relação entre a densidade aparente e a dureza normal às fibras - agrupamento dos valores médios para todas espécies. 
FIGURA 61 - Gráfico de ajuste de linha para a relação entre a densidade aparente e a tenacidade - agrupamento dos valores médios para todas espécies. 


\section{LISTA DE TABELAS}

TABELA 1 - Coeficientes de correlação $\quad 19$

TABELA 2 - Classes de resistência das dicotiledôneas. 56

TABELA 3 - Linearização de funções com transformações correspondentes $\quad 58$

TABELA 4 - Resultados da estatística de regressão para o agrupamento total das espécies. 63

TABELA 5 - Coeficientes obtidos para a regressão efetuada pelo agrupamento total de espécies. 64

TABELA 6 - Resultados da estatística de regressão para o agrupamento dos valores médios para todas espécies. $\quad 70$

TABELA 7 - Coeficientes obtidos para a regressão efetuada pelo agrupamento dos valores médios para todas espécies.

TABELA 8 - Valores de coeficientes de determinação para as propriedades analisadas - agrupamento total dos resultados. $\quad 77$

TABELA 9 - Valores de coeficientes de determinação para as propriedades analisadas - agrupamento médio dos resultados.

TABELA 10 - Apresentação das classes de resistência e resistências características para as espécies estudadas.

TABELA 11 - Apresentação dos resultados das resistências características das espécies.

TABELA 12 - Resultados das aferições das expressões utilizadas pela NBR 7190/1997 para caracterização simplificada da resistência da madeira.

TABELA 13 - Resultados obtidos para as resistências características utilizando as expressões da NBR 7190/1997. 
TABELA 14 - Resumo dos resultados da estatística de regressão para as relações estabelecidas.

TABELA 15 - Valores das propriedades de resistência e rigidez da madeira - Espécie: Votairea fusca.

TABELA 16 - Valores das propriedades de resistência e rigidez da madeira. Espécie: Vataireopsis araroba (Aguiar) Ducke.

TABELA 17 - Valores das propriedades de resistência e rigidez da madeira. Espécie: Hymenolobium sp.

TABELA 18 - Valores das propriedades de resistência e rigidez da madeira. Espécie: Dinizia excelsa Ducke.

TABELA 19 - Valores das propriedades de resistência e rigidez da madeira. Espécie: Hymenolobium petraeum Ducke.

TABELA 20 - Valores das propriedades de resistência e rigidez da madeira. Espécie: Votairea sp.

TABELA 21 - Valores das propriedades de resistência e rigidez da madeira. Espécie: Piptadenia macrocarpa Benth.

TABELA 22 - Valores das propriedades de resistência e rigidez da madeira. Espécie: Sebastiania commersoniana (Baill.) Smith \& Downs.

TABELA 23 - Valores das propriedades de resistência e rigidez da madeira. Espécie: Andira stipulacea Benth.

TABELA 24 - Valores das propriedades de resistência e rigidez da madeira. Espécie: Cassia ferruginea (Schrad.) S. Ex DC.

TABELA 25- Valores das propriedades de resistência e rigidez da madeira. Espécie: Ocotea odorifera (Vell.) Rohwer.

TABELA 26 - Valores das propriedades de resistência e rigidez da madeira. Espécie: Calycophyllum multiflorum Griseb.

TABELA 27 - Valores das propriedades de resistência e rigidez da madeira. Espécie: Calophyllum sp.

TABELA 28 - Valores das propriedades de resistência e rigidez da madeira. Espécie: Cedrela odorata Lin. 
TABELA 29 - Valores das propriedades de resistência e rigidez da madeira Espécie: Cedrela sp.

TABELA 30 - Valores das propriedades de resistência e rigidez da madeira. Espécie: Cedrelinga catenaeformis Ducke.

TABELA 31 - Valores das propriedades de resistência e rigidez da madeira. Espécie: Dipteryx odorata (Aubl.) Willd.

TABELA 32 - Valores das propriedades de resistência e rigidez da madeira. Espécie: Copaifera $c f$. ret.

TABELA 33 - Valores das propriedades de resistência e rigidez da madeira. Espécie: Goupia glabra Aubl.

TABELA 34 - Valores das propriedades de resistência e rigidez da madeira. Espécie: Goupia paraensis Hub.

TABELA 35 - Valores das propriedades de resistência e rigidez da madeira. Espécie: Apuleia leiocarpa (Vog.) Macbr.

TABELA 36 - Valores das propriedades de resistência e rigidez da madeira. Espécie: Planchonella pachycarpa Pires.

TABELA 37 - Valores das propriedades de resistência e rigidez da madeira. Espécie: Luetzelburgia sp.

TABELA 38 - Valores das propriedades de resistência e rigidez da madeira. Espécie: Peltophorum vogelianum Benth.

TABELA 39 - Valores das propriedades de resistência e rigidez da madeira. Espécie: Tabebuia serratifolia (Vahl) Nich.

TABELA 40 - Valores das propriedades de resistência e rigidez da madeira. Espécie: Mezilaurus itauba (Meissn.) Taub.

TABELA 41 - Valores das propriedades de resistência e rigidez da madeira. Espécie: Hymenaea sp (Hayne) Lee et Lang.

TABELA 42 - Valores das propriedades de resistência e rigidez da madeira. Espécie: Ocotea sp.

TABELA 43 - Valores das propriedades de resistência e rigidez da madeira. Espécie: Manilkara huberi (Ducke) Standl.

TABELA 44 - Valores das propriedades de resistência e rigidez da madeira. Espécie: Qualea paraensis Ducke. 
TABELA 45 - Valores das propriedades de resistência e rigidez da madeira. Espécie: Clarisia racemosa R.\& Pav.

TABELA 46 - Valores das propriedades de resistência e rigidez da madeira. Espécie: Prodosia sp.

TABELA 47 - Valores das propriedades de resistência e rigidez da madeira. Espécie: Parinari excelsa Sabine.

TABELA 48 - Valores das propriedades de resistência e rigidez da madeira. Espécie: Tapirira sp.

TABELA 49 - Valores das propriedades de resistência e rigidez da madeira. Espécie: Erisma uncinatum Warm.

TABELA 50 - Valores das propriedades de resistência e rigidez da madeira. Espécie: Vochysia haenkeana (Spreng.) Mart.

TABELA 51 - Valores das propriedades de resistência e rigidez da madeira. Espécie: Diplotropis incexis Rizz. \& Matt.

TABELA 52 - Valores das propriedades de resistência e rigidez da madeira. Espécie: Tachigali myrmecophila Ducke.

TABELA 53 - Valores das propriedades de resistência e rigidez da madeira. Espécie: Bagassa guianensis Aubl.

TABELA 54 - Valores das propriedades de resistência e rigidez da madeira. Espécie: Qualea retusa. 


\title{
LISTA DE ABREVIATURAS E SIGLAS
}

\author{
ABNT - $\quad$ Associação Brasileira de Normas Técnicas \\ FPL - $\quad$ Forest Products Laboratory \\ IBAMA - Instituto Brasileiro do Meio Ambiente e dos Recursos Naturais \\ Renováveis \\ INPA - $\quad$ Instituto Nacional de Pesquisa da Amazônia \\ IPT - Instituto de Pesquisas Tecnológicas \\ LaMEM - $\quad$ Laboratório de Madeiras e Estruturas de Madeiras \\ NBR - $\quad$ Norma Brasileira Registrada \\ PSF - $\quad$ Ponto de Saturação das Fibras \\ RFF - $\quad$ Rede Ferroviária Federal \\ SENAI - $\quad$ Serviço de Aprendizagem Comercial \\ TRADA - Timber Research \& Development Association
}




\section{LISTA DE SÍMBOLOS}

$\mathrm{a}, \mathrm{b}$

d

$\bar{d}$

$\mathrm{E}_{\mathrm{c} 0}$

$\mathrm{E}_{\mathrm{c} 0, \mathrm{~m}}$

$\mathrm{E}_{\mathrm{M} 0}$

$\mathrm{E}_{\mathrm{t} 0}$

$\mathrm{E}_{\mathrm{U} \%}$

$\mathrm{E}_{12}$

$\mathrm{f}_{\mathrm{c} 0}$

$\mathrm{f}_{\mathrm{c} 0, \mathrm{k}}$

$\mathrm{f}_{\mathrm{H} 0}$

$\mathrm{f}_{\mathrm{H} 90}$

$f_{M}$

$\mathrm{f}_{\mathrm{S} 0}$

$\mathrm{f}_{\mathrm{t} 0}$

$f_{t 0, k}$

$\mathrm{f}_{\mathrm{t} 90}$

$\mathrm{f}_{\mathrm{tM}, \mathrm{k}}$

$\mathrm{f}_{\mathrm{U} \%}$

$\mathrm{f}_{\mathrm{V} 0 \mathrm{c}}$

$\mathrm{f}_{\mathrm{vk}}$

$f_{\mathrm{w}, \mathrm{k}}$

$\mathrm{f}_{12}$

$\mathrm{m}_{\mathrm{s}}$
- constantes (coeficientes da regressão);

- desvios (variável estudada no teste "pairing");

- estimativa da média dos desvios

- módulo de elasticidade na compressão paralela às fibras;

- módulo de elasticidade médio na compressão paralela às fibras;

- módulo de elasticidade na flexão estática;

- módulo de elasticidade na tração paralela às fibras;

- módulo de elasticidade para a umidade U\%;

- módulo de elasticidade corrigido para umidade de 12\%;

- resistência à compressão paralela às fibras;

- resistência característica à compressão paralela às fibras;

- dureza paralela às fibras;

- dureza normal às fibras;

- resistência convencional no ensaio de flexão estática;

- resistência ao fendilhamento;

- resistência à tração paralela às fibras;

- resistência característica da madeira `a tração paralela às fibras;

- resistência à tração normal às fibras;

- resistência característica convencional no ensaio de flexão estática;

- resistência para a umidade U\%;

- resistência ao cisalhamento;

- resistência característica ao cisalhamento;

- resistência característica;

- resistência corrigida para a umidade de $12 \%$;

- massa seca da amostra; 
$\mathrm{m}_{12} \quad$ - massa da madeira a $12 \%$ de umidade;

N $\quad$ - Número de amostras;

p - Valor $\mathrm{p}$, probabilidade de t exceder numericamente o valor tabelado

(notação utilizada para análise da regressão);

$\mathrm{R}^{2} \quad$ - coeficiente de determinação;

S - erro padrão de estimativa para a regressão;

$\mathrm{S}_{\mathrm{d}} \quad$ - desvio padrão da amostra;

SE - erro padrão de estimativa das variáveis;

- variável t de Student, valor estatístico calculado (notação utilizada para análise da regressão);

$\mathrm{t}_{\phi, 95 \%} \quad$ - valor tabelado, para $\phi$ graus de liberdade e $95 \%$ de probabilidade;

$\mathrm{T} \quad$ - tenacidade;

$\mathrm{U} \% \quad$ teor de umidade;

$\mathrm{V}_{\text {sat }} \quad$ - volume da amostra saturada;

$\mathrm{V}_{\text {seca }} \quad$ - volume do corpo-de-prova, para a madeira seca;

$\mathrm{V}_{\mathrm{U} \%} \quad$ - volume do corpo-de-prova, ao teor de umidade $\mathrm{U} \%$;

$\mathrm{V}_{12} \quad$ - volume da madeira a $12 \%$ de umidade;

$\Delta \mathrm{V} \quad$ - retração volumétrica, para a variação de umidade entre $\mathrm{U} \%$ e $0 \%$;

$\rho_{\text {bas }} \quad$ - densidade básica;

$\rho_{\text {apar }} \quad$ - densidade aparente;

$\delta_{\mathrm{V} \%} \quad$ - coeficiente de retratibilidade volumétrica;

$\rho_{\mathrm{u} \%} \quad$ - densidade aparente, ao teor de umidade U\%;

$\rho_{12} \quad$ - densidade aparente, ao teor de umidade $12 \%$;

$\varepsilon_{\mathrm{r}, 2} \quad$ - retratibilidade radial total;

$\varepsilon_{\mathrm{r}, 3} \quad-$ retratibilidade tangencial total;

$\frac{\mathrm{S}_{\mathrm{d}}}{\sqrt{\mathrm{n}}} \quad$ - erro padrão de estimativa;

$\phi \quad$ - número de graus de liberdade;

$\mu_{\mathrm{d}} \quad$ - média dos desvios. 


\section{RESUMO}

DIAS, F. M. (2000). A densidade aparente como estimador de propriedades de resistência e rigidez da madeira. São Carlos, 138p. Dissertação (Mestrado) -, Área Interunidades - Instituto de Física de São Carlos, Instituto de Química de São Carlos, Escola de Engenharia de São Carlos - Universidade de São Paulo.

Neste trabalho são determinadas as correlações entre a densidade aparente e as propriedades físicas, de resistência e de rigidez da madeira, de quarenta espécies nativas brasileiras, do grupo dicotiledôneas. Através de análise estatística, foram obtidas expressões matemáticas que permitem estimar, a partir da densidade aparente, grande parte das propriedades de resistência e rigidez da madeira. Tais expressões são apresentadas como proposta para a utilização na caracterização de espécies menos conhecidas, o que viabiliza o adequado emprego de espécies nativas nas mais variadas aplicações para as quais a madeira é indicada. Apresentam-se também aferições das expressões propostas pela norma brasileira NBR 7190, que permitem a caracterização simplificada das resistências da madeira de espécies usuais a partir dos ensaios de resistência à compressão paralela às fibras.

Palavras-chave: madeira; densidade aparente; resistência; rigidez. 


\begin{abstract}
In this study the correlations between the specific gravity, physical and mechanical properties of wood from forty Brazilian native species of hardwoods are determined. The mathematical expressions obtained through this study, permit estimate most wood properties of strength and stiffness, based on their specific gravity. Such expressions are proposed as means of characterizing less-known species, what makes possible the appropriate employment of native species in most varied applications for which wood is the indicated material. Furthermore, it is presented the calibration of the expressions proposed by the Brazilian code NBR 7190 , being these a simplified method of characterizing mechanical properties of common species of wood by means of tests of strength in compression parallel to the grain.
\end{abstract}

Keywords: wood; specific gravity; strength; stiffness. 